\title{
BMJ Open Interventions to integrate care for people with serious mental illness and substance use disorders: a systematic scoping review protocol
}

\author{
Amy Richardson (1) , ${ }^{1}$ Lauralie Richard, ${ }^{2}$ Kathryn Gunter, ${ }^{3}$ Sarah Derrett ${ }^{1}$
}

To cite: Richardson A, Richard L, Gunter K, et al. Interventions to integrate care for people with serious mental illness and substance use disorders: a systematic scoping review protocol. BMJ Open 2019;9:e031122. doi:10.1136/ bmjopen-2019-031122

- Prepublication history for this paper is available online. To view these files, please visit the journal online (http://dx.doi org/10.1136/bmjopen-2019031122).

Received 17 April 2019

Revised 26 September 2019

Accepted 10 October 2019

Check for updates

(c) Author(s) (or their employer(s)) 2019. Re-use permitted under CC BY-NC. No commercial re-use. See rights and permissions. Published by BMJ.

${ }^{1}$ Department of Preventive and Social Medicine, Dunedin School of Medicine, University of Otago, Dunedin, New Zealand

${ }^{2}$ Department of General Practice \& Rural Health, Dunedin School of Medicine, University of Otago, Dunedin, New Zealand

${ }^{3}$ Chicago Center for Diabetes Translation Research,

The University of Chicago, Department of Medicine, Chicago, Illinois, USA

Correspondence to Dr Amy Richardson; amy.richardson@otago.ac.nz

\section{ABSTRACT}

Introduction People with serious mental illness (SMI) and/or substance use disorders (SUDs) have an elevated risk of premature mortality compared with the general population. This has been attributed to higher rates of chronic illness among these individuals, but also to inequities in healthcare access and treatment. Integrated care has the potential to improve the health of people with SMI/SUDs. The aims of this scoping review are to: (1) identify empirical investigations of interventions designed to integrate care for people with SMI/SUDs; (2) describe the underlying theories, models and frameworks of integrated care that informed their development; and (3) determine the degree to which interventions address dimensions of a comprehensive and validated framework of integrated care.

Methods and analysis Guidelines for best practice and reporting of scoping reviews will be followed using the framework of Arksey and O'Malley and the Preferred Reporting Items for Systematic Reviews and MetaAnalyses scoping review checklist. An iterative and systematic search of peer-reviewed publications reporting empirical research findings will be conducted. This literature will be identified by searching five databases: Medline (Ovid), PsycINF0, CINAHL, Embase (Ovid) and Scopus. The search will be restricted to articles published between January 2000 and April 2019. Two reviewers will independently screen publications in two successive stages of title and abstract screening, followed by full-text screening of eligible publications. A tabular summary and narrative synthesis will be completed using data extracted from each included study. A framework synthesis will also be conducted, with descriptions of interventions mapped against a theoretical framework of integrated care.

Ethics and dissemination This review will identify the extent and nature of empirical investigations evaluating interventions to integrate care for people with SMI/SUDs. Ethical approval was not required. A team of relevant stakeholders, including people with lived experience of mental health conditions, has been established. This team will be engaged throughout the review and will ensure that the findings are widely disseminated. Dissemination will include publication of the review in a peer-reviewed journal. The review protocol has been registered through Open Science Framework and can be accessed at https:// osf.io/njkph/
Strengths and limitations of this study

- This systematic scoping review will identify the extent and nature of empirical investigations evaluating interventions to integrate care for people with serious mental illness and/or substance use disorders.

- The review will reveal which aspects of integrated care have been targeted in interventions to date and highlight potential opportunities for future research.

- A comprehensive team of relevant stakeholders, including people with lived experience of mental health conditions, mental health professionals, other health professionals and researchers from a range of disciplines, will be involved in all stages of the review process.

- The review will be restricted to articles published in English and this may prevent a number of integrated care interventions from being detected.

- No quality appraisal of included studies will be completed, precluding conclusions about the effectiveness of different integrated care approaches at improving outcomes.

\section{BACKGROUND}

Serious mental illness (SMI; also referred to as severe and enduring mental illness or SEMI) includes a range of conditions, such as major depression, bipolar disorder and schizophrenia. ${ }^{12}$ These conditions are associated with debilitating symptoms that require ongoing treatment or management. People with SMI have a significantly reduced life expectancy and are at risk of poor health outcomes relative to those in the general population. $^{3}$ In New Zealand, men and women using mental health services have more than twice the risk of experiencing premature mortality than the general population. ${ }^{4}$ This is similar to the UK, where a recent study of a nationally representative cohort of people with bipolar disorder and schizophrenia found that the rate of allcause mortality was 1.77 times greater among 
individuals with bipolar disorder and 2.08 times greater for individuals with schizophrenia. ${ }^{5}$ The UK study also found that these disparities in mortality had increased significantly from the year 2000 to $2014 .^{5}$

Evidence suggests that people with substance use disorders (SUDs) are also at increased risk of mortality compared with the general population. These disorders reflect the pattern of symptoms that result from prolonged use of illicit or legal drugs, including alcohol and medicines, despite mental or physical problems associated with their use. ${ }^{6}$ The reduced life expectancy associated with SUDs is estimated to be 13.8 years, higher than the 6.3year reduction associated with depression and the 7.2year reduction associated with schizophrenia. ${ }^{7}$ Of particular concern is the high prevalence of co-occurring SMI and SUDs. ${ }^{8}$ A systematic review of studies conducted in the UK found the prevalence of co-occurring SMI and SUDs to be between $0.05 \%-0.16 \%$ in the general population. ${ }^{9}$ In contrast, current harmful drug use or dependence among people with SMI was $1.9 \%-$ $7.0 \%$, and current harmful alcohol use or dependence was $7.0 \%-15.5 \% .^{9}$

The lower average life expectancy evident among people with SMI/SUDs is largely attributable to an increased risk of a number of chronic health conditions. ${ }^{10}$ Cardiovascular diseases have been identified as the most common cause of death in the SMI population, ${ }^{11} 12$ contributing to more than $30 \%$ of all deaths among public mental health clients across eight US states between 1997 and $2000 .{ }^{12}$ This contrasts to the percentage of deaths due to suicide over the same time period, which did not exceed $15 \%$ in any state, during any year examined. ${ }^{12}$ Metabolic syndrome has been found to affect as many as one in three people with SMI, ${ }^{13}$ and type 2 diabetes occurs at almost twice the rate among people with SMI than the general population. ${ }^{14}$ While the incidence of cancer is no greater in people with SMI than in the general population, these individuals are more likely to have metastases at diagnosis and are less likely to receive specialist cancer treatment resulting in higher cancer mortality rates. ${ }^{15} 16$ Similarly, after adjusting for age and gender, people with SUDs have been identified as at increased risk of diabetes, heart disease, asthma, gastrointestinal disorders, skin infections, malignant neoplasms and acute respiratory disorders. ${ }^{17}$ However, risk of these disorders is substantially greater for individuals with comorbid SMI and SUDs, particularly individuals with psychosis. ${ }^{17}$ There is growing acknowledgement that people with these comorbid conditions experience the worst health, well-being and social outcomes, and are among the most disadvantaged and vulnerable in society. ${ }^{9}$

A number of factors have been found to contribute to the high prevalence of chronic health conditions which, in turn, contribute to reduced life expectancy among people with SMI/SUDs. These include socioeconomic disadvantage,${ }^{18}$ obesity and poor nutrition, ${ }^{19}$ reduced physical activity, ${ }^{20}{ }^{21}$ side-effects of antipsychotic medication, ${ }^{10}$ elevated consumption of alcohol and illicit drugs ${ }^{22}$ and high rates of smoking. ${ }^{2324}$ However, there is increasing evidence that the poor health outcomes among people with SMI/SUDs are also a result of inequities in the provision of healthcare. ${ }^{25}{ }^{26}$ In addition, difficulties with access to healthcare or routine screening among people with SMI/SUDs have been identified. ${ }^{27}$ Even when healthcare is accessed, these individuals have been found to receive poorer quality care, as well as higher rates of misdiagnosis, and lower rates of specialist interventions that could prevent the progression of a number of diseases, ${ }^{1028}$ compared with people without SMI/SUDs. Stigma has a pervasive influence on the quality of care that is provided to people with SMI/SUDs, ${ }^{25}$ with medical professionals frequently disregarding the physical health concerns of this population and misinterpreting physical symptoms as mental illness. ${ }^{29}$

One strategy to address inequities in healthcare access and treatment for people with SMI/SUDs is the integration of healthcare and social services. Integration of care is increasingly recognised as the most appropriate method for delivering care to people with multiple, complex chronic conditions, and has been found to be associated with significant improvements in conditionspecific quality of life. ${ }^{30}$ However, a consensus on the concept of integrated care is yet to be reached, presenting difficulties for meaningful evaluation of integrated care approaches. ${ }^{31}{ }^{32}$ Some definitions are process oriented, some (although few) are person-centred, and others are health service oriented. ${ }^{33}$ In an effort to provide a comprehensive concept of integrated care, Singer et al developed an integrated care framework that emphasises the importance of both care-coordination and person-centred care, acknowledging the central role of service users/ patients and their families in the management of their own health. ${ }^{34}$ They describe integrated care as: "patient care that is coordinated across professionals, facilities and support systems; continuous over time and between visits; tailored to the patients' needs and preferences; and based on shared responsibility between patient and caregivers for optimising health' (Singer et al, p 113). ${ }^{34}$ Because of the varied definitions of integrated care, it is important to understand the underlying theories, models or frameworks of integrated care that are being used to inform empirical research in this area.

In the mental health context, a number of strategies to integrate care have been investigated. ${ }^{35}{ }^{36}$ Examples of intervention strategies include the co-location of mental and physical health services within a single setting, ${ }^{37-39}$ collaborative care meetings between general practitioners and mental health professionals, ${ }^{40}$ and the appointment of case managers to liaise between services and coordinate the overall care of individuals with SMI. ${ }^{41}{ }^{42}$ Interventions for people with co-occurring mental and addictive disorders have also been explored, such as on-site medical consultations, team-based approaches and facilitated referrals to primary care ${ }^{26}$ Despite substantial research in this area, the number and types of integrated care interventions that have been investigated empirically among 
each population is unknown. It is also unclear which outcomes have been examined in evaluations of interventions aiming to integrate care (eg, whether the goal has been to increase contact with healthcare professionals or to improve the physical health of mental health/addiction service users). Most importantly, the underlying theoretical models on which these interventions have been based are yet to be identified.

These gaps in evidence suggest that a scoping of the literature could help to identify the characteristics of interventions that have integrated care for people with SMI/SUDs to date. While these individuals represent groups with distinct diagnoses, the symptom burden associated with the diagnoses is highly similar, ${ }^{43}$ and they frequently co-occur. ${ }^{44}$ Both groups also face barriers to receiving integrated care that could lead to more timely and effective treatment of physical health conditions. ${ }^{45}$

Scoping reviews are recommended to examine the extent, range and nature of the evidence relating to a topic, providing an opportunity to clarify concepts, identify knowledge gaps and inform future research, practice and policy-making. ${ }^{46}$ We intend to identify the types of empirically tested interventions aiming to integrated care for people with SMI/SUDs that have been investigated; the range of outcomes these investigations have endeavoured to modify; the theories, models and frameworks of integrated care that have informed intervention development; and the extent to which interventions have addressed key components of a widely recognised framework for the delivery of integrated care. ${ }^{34}$ Given the significance of the inequities in health and mortality for people with SMI/SUDs, an understanding of the degree to which interventions to integrate care for this population are meeting key components of successful integrated care delivery is extremely important.

\section{OBJECTIVES}

The aims of the proposed scoping review are to: (1) systematically identify and describe empirical investigations of interventions to integrate care for people with SMI/SUDs, (2) describe the theories/models/frameworks of integrated care informing the empirical research and (3) determine the degree to which identified interventions address components of a comprehensive and validated framework of integrated care.

\section{METHODS}

This scoping review will be conducted according to the methods developed by Arksey and O'Malley, ${ }^{48}$ and the subsequent refinements to these methods. ${ }^{49} 50$ There are six steps including: (1) defining the research question/s; (2) identifying relevant studies; (3) study selection; (4) charting the data; (5) collating, summarising and reporting the results; and (6) consultation. Preferred Reporting Items for Systematic Reviews and Meta-Analyses (PRISMA) guidelines will be followed using the PRISMA extension for scoping reviews checklist. ${ }^{46}$ An iterative approach will be taken toward searching the literature, refining the search strategy, reviewing articles for inclusion and extracting relevant data. The review protocol has been registered through Open Science Framework and can be accessed at: https://osf.io/njkph/. Any amendments or deviations from the protocol will be reported in the methods section of the final published review.

\section{Defining the research question}

Research questions were formulated by considering the concept (integrated care), target population (people with SMI/SUDs), context (healthcare settings) and outcomes (empirically investigated outcomes) of interest in order to clarify the focus of the review and establish an effective search strategy. This scoping review intends to answer the following research questions:

1. What types of interventions have been designed to integrate care for people with SMI/SUDs across a broad range of healthcare settings, and which have been associated with improvements in outcomes?

2. What outcomes have studies examining interventions to integrate care for people with SMI/SUDs sought to modify?

3. Which theories, models or frameworks of integrated care have been used to inform intervention development?

4. Which components of an existing comprehensive and validated framework (Framework for Measuring Integrated Patient Care $)^{34}$ have been addressed by interventions to integrate care for people with SMI/ SUDs?

\section{Identifying relevant studies}

Our search strategy was developed with the goal of undertaking a comprehensive review of the existing evidence base. An experienced subject librarian at the University of Otago has been consulted to assist with the identification of relevant search terms and databases. Search terms have also been reviewed by a team of relevant stakeholders, including people with lived experience of mental health conditions, mental health professionals, other health professionals and researchers from a range of disciplines.

In order to identify empirical literature, an initial limited search of a selection of relevant databases has been performed followed by a review of text words contained in the titles and abstracts, and of index terms used to describe the articles. A second search will be conducted using all identified keywords and index terms and will be undertaken across five databases: Medline (Ovid), PsycINFO, CINAHL, Embase (Ovid) and Scopus. The reference lists of all included articles will be searched for additional studies. The search will be restricted to articles and reports published in English and to articles published between January 2000 and April 2019. The search strategy has been developed in Medline (Ovid) and will be adapted to other databases (see table 1). All searches will include a combination of subject headings, 


\begin{tabular}{|c|c|c|}
\hline $\begin{array}{l}\text { Line } \\
\text { number }\end{array}$ & $\begin{array}{l}\text { Search term entered into Ovid } \\
\text { (Medline) }\end{array}$ & Results \\
\hline 1 & intervention.mp. & 464809 \\
\hline 2 & $\begin{array}{l}\text { Program Evaluation/ or Evaluation } \\
\text { Studies/ or evaluation.mp. }\end{array}$ & 1404582 \\
\hline 3 & program.mp. or Programs/ & 411198 \\
\hline 4 & programme.mp & 79501 \\
\hline 5 & 1 or 2 or 3 or 4 & 2132570 \\
\hline 6 & $\begin{array}{l}\text { Primary Health Care/ or "Delivery } \\
\text { of Health Care, Integrated"/ or } \\
\text { integrated care.mp. }\end{array}$ & 83223 \\
\hline 7 & integrated services.mp. & 516 \\
\hline 8 & integrated health.mp. & 2604 \\
\hline 9 & integration of care.mp. & 280 \\
\hline 10 & integration of services.mp. & 294 \\
\hline 11 & integrating care.mp. & 119 \\
\hline 12 & care integration.mp. & 320 \\
\hline 13 & collaborative care.mp. & 1681 \\
\hline 14 & $\begin{array}{l}\text { Patient Care Team/ or coordinated } \\
\text { care.mp. }\end{array}$ & 63179 \\
\hline 15 & $\begin{array}{l}6 \text { or } 7 \text { or } 8 \text { or } 9 \text { or } 10 \text { or } 11 \text { or } 12 \text { or } \\
13 \text { or } 14\end{array}$ & 145318 \\
\hline 16 & $\begin{array}{l}\text { (severe and enduring mental illness). } \\
\mathrm{mp} \text {. }\end{array}$ & 48 \\
\hline 17 & serious mental illness.mp & 2474 \\
\hline 18 & $\begin{array}{l}\text { Mental Disorders/ or serious mental } \\
\text { disorder.mp. }\end{array}$ & 155387 \\
\hline 19 & serious psychiatric illness & 53 \\
\hline 20 & serious psychiatric disorder & 83 \\
\hline 21 & severe mental illness & 3208 \\
\hline 22 & severe mental disorder & 282 \\
\hline 23 & severe psychiatric illness & 106 \\
\hline 24 & severe psychiatric disorder & 159 \\
\hline 25 & $\begin{array}{l}\text { Schizophrenia, Catatonic/ or } \\
\text { Schizophrenia, Disorganized/ } \\
\text { or Schizophrenia, Paranoid/ or } \\
\text { schizophrenia.mp. }\end{array}$ & 123422 \\
\hline 26 & $\begin{array}{l}\text { psychosis.mp. or Psychotic } \\
\text { Disorders/ }\end{array}$ & 58808 \\
\hline 27 & $\begin{array}{l}\text { bipolar disorder.mp. or Bipolar } \\
\text { Disorder/ }\end{array}$ & 42908 \\
\hline 28 & $\begin{array}{l}\text { Depressive Disorder, Major/ or major } \\
\text { depression.mp. }\end{array}$ & 39682 \\
\hline 29 & Substance-Related Disorders/ & 91316 \\
\hline 30 & $\begin{array}{l}16 \text { or } 17 \text { or } 18 \text { or } 19 \text { or } 20 \text { or } 21 \text { or } 22 \\
\text { or } 23 \text { or } 24 \text { or } 25 \text { or } 26 \text { or } 27 \text { or } 28 \text { or } \\
29\end{array}$ & 434086 \\
\hline 31 & 5 and 15 and 30 & 2979 \\
\hline
\end{tabular}

Run date=17 April 2019. related terms and keywords. Boolean logic and operators (ie, 'and', 'or') will be used to combine and refine search terms and concepts.

\section{Study selection}

All records retrieved from the searches will be exported to Endnote referencing database. Following this, duplicate records will be removed (using both the Endnote 'de-duping' function and a manual scan of records), and the number of unique records will be identified.

A two-stage collaborative review process will select studies for inclusion. Screening of studies will be piloted by two reviewers (AR and LR) on the first $5 \%$ of citations retrieved from the database search to test eligibility criteria and reviewer agreement. After consensus on each of these citations is reached, the reviewers will independently apply eligibility criteria during the initial title/ abstract review. Titles and abstracts will be retained for full text review if they: (1) refer to an intervention to integrate care; (2) the intervention is for people with mental health conditions, people with substance use problems or health professionals responsible for their care; and (3) the intervention is set in a health-oriented context. The full text of relevant studies will then be obtained and independently assessed for eligibility by two reviewers (AR and LR). After each review stage, the reviewer's agreement will be assessed and a third reviewer (SD) will be consulted in cases of disagreement, until consensus is achieved.

Eligibility criteria for a full text article to be included have been developed a priori with the assistance of the stakeholder team. This criteria is identified below in relation to participants, interventions, outcomes, context and study design.

\section{Participants}

Populations of interest will include: (1) adults with SMI/ SUDs who have received an intervention designed to integrate care or (2) healthcare professionals or associated staff (including unregistered health workers, managers and administrators; hereafter referred to collectively as 'health providers') who were involved in the delivery of an intervention to integrate care for people with SMI/SUDs. In the present investigation, SMI is defined as mental illnesses (schizophrenia, schizoaffective disorder, bipolar disorder, major depression and other psychoses) that produce severe and debilitating symptoms for 12 months or more. ${ }^{251}$ Following feedback from our stakeholder group, and the widely recognised challenges associated with integrating care for people with SUDs, the decision was made to also review interventions for this population. SUDs is defined problems resulting from alcohol or other drug use for 12 months or more. ${ }^{6}$ Despite facing many of the same health and mortality burdens as people with SMI, as well as inequities in access to appropriate care, this population is frequently overlooked in the development and evaluation of clinically integrated service delivery approaches. ${ }^{45}$ 


\section{Interventions}

Studies and reports describing interventions (ie, activities, programmes or strategies) with the explicit goal of integrating care for people with SMI/SUDs, addressing any of the key components of integrated care defined by Singer et $a l,{ }^{34}$ will be eligible for inclusion. This includes studies endeavouring to integrate care both within and between organisations and services. Eligible integrated care interventions can be very specific or can be implemented across a broad range of domains (ie, funding, administrative, organisational, service delivery and clinical domains). ${ }^{52}$

\section{Outcomes}

A broad range of service user and provider outcomes will be included in order to identify which outcomes have been most frequently examined. However, primary outcomes of interest will be service user health behaviours and physical health outcomes, given the potential of integrated care to increase access to treatments designed to improve physical health. Examples of secondary outcomes for consideration include: cost-effectiveness, patterns of healthcare utilisation and perceived satisfaction with an intervention (from service user and/or provider perspectives). Studies investigating process-oriented indicators and evaluation outcomes will be excluded, as the focus of this scoping review is on identifying the specific outcomes integrated care approaches are endeavouring to improve.

\section{Context}

Studies and reports published between January 2000 and May 2019 will be eligible for inclusion. This time period was selected to ensure identification of interventions likely to be relevant and applicable to contemporary healthcare contexts. Interventions delivered in any healthcare settings will be eligible, including primary care and community care settings, forensic settings, outpatient clinics, acute care hospitals and long-term care facilities.

\section{Study Design}

All empirical investigations examining outcomes following the implementation of an integrated care intervention using quantitative, qualitative or mixed methods designs will be eligible for inclusion. Quantitative studies will include randomised and non-randomised controlled trials, as well as studies implementing before-after designs (with or without a control group), and crosssectional studies. Qualitative investigations of participants' perceptions or experiences of an intervention will also be considered, including (but not limited to) designs such as qualitative description, phenomenology, grounded theory, ethnography and action research. Pilot studies will be included, whereas conceptual articles will be excluded, in addition to those reporting case study and quality improvement designs.

\section{Data extraction}

Data will be extracted according to the recommendations of Arksey and O'Malley. ${ }^{48}$ A standardised extraction excel spreadsheet will be used to record: author(s), year of publication, study location, intervention type (and any comparator), underlying theory of integrated care, duration of the intervention, study population, aims of the study, methods, outcomes and key findings. Data extraction will be performed independently by two researchers (AR and $K G$ ), and compared by a third researcher $(\mathrm{SD})$. The third researcher will be consulted to resolve any discrepancies in data extraction relating to each study. Possible additions/modifications to the data extraction form may be made after review of the first five references in order to ensure that all relevant information will be captured.

\section{Collating, summarising and reporting}

A two-step approach will be used to summarise the findings of included studies. Step one will involve a narrative synthesis of the characteristics and findings of the studies (including tabular and/or graphical summaries). Studies will be organised according to intervention type in order to highlight the range of integrated care approaches for people with SMI/SUDs that have been empirically evaluated. The underlying theory of integrated care associated with each included intervention will also be described (where this information is available).

Step two will identify the degree to which interventions for people with SMI/SUDs have addressed dimensions of integrated care as conceptualised by Singer et $a l .{ }^{34} \mathrm{~A}$ framework synthesis will be conducted to review the included interventions, with coding and analysis directed by the integrated care framework. Specifically, we are interested in qualitatively analysing the extent to which each intervention description addresses the seven elements of integrated care: (1) coordination within a care team, (2) coordination across care teams, (3) coordination between care teams and community resources, (4) continuous familiarity with patients over time, (5) continuous proactive and responsive action between visits, (6) service user-centred care and (7) shared responsibility. ${ }^{34}$ To do so, descriptions of the interventions will be imported to an excel spreadsheet and analysed by two authors (AR and LR); both researchers have previous experience coding qualitative data. The a priori coding framework will be applied to each intervention description independently by the researchers. Results from these analyses will be summarised in order to highlight dimensions of integrated care that require further investigation and implementation among people with SMI/SUDs.

\section{Consultation process}

The aim of this review is to identify and describe empirical investigations of interventions to integrate care for people with SMI/SUDs in order to highlight which interventions have been associated with positive outcomes and which dimensions of integrated care have been targeted. This information will have potential to inform both future research activity and clinical practice. In order to ensure that findings of the review are of relevance to 
mental health and addiction service users, and those who provide care to these individuals, we have engaged a stakeholder team as mentioned above. Stakeholders have been involved in developing the research questions guiding this review and have reviewed the search strategy to identify key terms that are relevant to the population, concept and contexts of interest presented in this protocol. Stakeholders will also be involved in interpreting the review findings and will advise on dissemination.

\section{Patient and public involvement}

This scoping review protocol has engaged the expertise of individuals with lived experience of SMI. These individuals have contributed to the development of the research questions, reviewed and made suggestions to the proposed search terms, and will be extensively involved during the interpretation and dissemination phases of this project.

\section{ETHICS AND DISSEMINATION}

Although integrated care is increasingly recommended for people with SMI/SUDs, it is unclear what elements of integrated care have been investigated in empirical evaluations of interventions designed to improve outcomes for these populations. To our knowledge, our scoping review will be the first to systematically describe the extent and nature of interventions to integrate care for people with SMI and people with SUDs, including which outcomes these interventions have endeavoured to modify. Therefore, the scoping review findings are expected to be of interest to service users, researchers, clinicians and policymakers. Our dissemination strategy will include publication of the review in an open-access peer-reviewed journal (i.e., available to service users, their families and the general public), and scientific presentations of the findings at conferences and to staff working within a range of mental health and addiction settings. All stakeholders will be involved in interpreting the review findings and ensuring that these are widely disseminated through their respective networks-including to service users. This will be facilitated by a half-day round-table meeting with our stakeholder group. During this meeting, findings of the review will be discussed and opportunities for future areas of research and clinical practice work will be brainstormed. It is hoped that stakeholders' knowledge and interpretations of the review findings will identify clear priorities for changes in the development and delivery of integrated care.

Acknowledgements We would like to acknowledge Christy Ballard, the subject librarian for the Department of Preventive and Social Medicine at the University of Otago for her assistance with developing a preliminary search strategy. We would also like to acknowledge the members of our stakeholder group who helped to develop the research questions guiding the proposed review and who also provided suggestions regarding additional relevant search terms. Stakeholder group members include: Dr Emma Wyeth, Professor Tim Stokes, Ms Mel Green, Ms Adell Cox, Mr Martin Burke, Mr Shaun McNeil, Dr Ruth Cunningham, Mrs Helen Lockett and Dr Helen Hamer.
Contributors AR completed an initial draft of the review protocol. LR assisted with development of the research questions and eligibility criteria. KG helped to develop the methods for article screening and data extraction. SD led the conceptualisation of the review. All authors have provided feedback on multiple iterations of the manuscript.

Funding This research is funded by a University of Otago Research Grant (NZD \$34743).

Competing interests None declared.

Patient consent for publication Not required.

Provenance and peer review Not commissioned; externally peer reviewed.

Open access This is an open access article distributed in accordance with the Creative Commons Attribution Non Commercial (CC BY-NC 4.0) license, which permits others to distribute, remix, adapt, build upon this work non-commercially, and license their derivative works on different terms, provided the original work is properly cited, appropriate credit is given, any changes made indicated, and the use is non-commercial. See: http://creativecommons.org/licenses/by-nc/4.0/.

ORCID iD

Amy Richardson http://orcid.org/0000-0003-4776-3059

\section{REFERENCES}

1 Ruggeri M, Leese M, Thornicroft G, et al. Definition and prevalence of severe and persistent mental illness. Br J Psychiatry 2000;177:149-55.

2 Kronenberg C, Doran T, Goddard M, et al. Identifying primary care quality indicators for people with serious mental illness: a systematic review. Br J Gen Pract 2017;67:e519-30.

3 Ilyas A, Chesney E, Patel R. Improving life expectancy in people with serious mental illness: should we place more emphasis on primary prevention? Br J Psychiatry 2017;211:194-7.

4 Cunningham R, Sarfati D, Peterson D, et al. Premature mortality in adults using new Zealand psychiatric services. N Z Med J 2014;127.

5 Hayes JF, Marston L, Walters K, et al. Mortality gap for people with bipolar disorder and schizophrenia: UK-based cohort study 2000-2014. Br J Psychiatry 2017;211:175-81.

6 Hasin DS, O'Brien CP, Auriacombe M, et al. Dsm-5 criteria for substance use disorders: recommendations and rationale. AJP 2013;170:834-51.

7 Hannerz H, Borga P, Borritz M. Life expectancies for individuals with psychiatric diagnoses. Public Health 2001;115:328-37.

8 Buckley PF. Prevalence and consequences of the dual diagnosis of substance abuse and severe mental illness. J Clin Psychiatry 2006;67.

9 Megnin-Viggars O, Brown M, Marcus E, et al. The epidemiology, and current configuration of health and social care community services, for people in the UK with a severe mental illness who also misuse substances. prepared for the public health internal guidelines, centre for guidelines at the National Institute for health and care excellence (NICE. National Collaborating Centre for Mental Health, 2016.

10 DE HERT M, Correll CU, Bobes J, et al. Physical illness in patients with severe mental disorders. I. prevalence, impact of medications and disparities in health care. World Psychiatry 2011;10:52-77.

11 De Hert M, Dekker JM, Wood D, et al. Cardiovascular disease and diabetes in people with severe mental illness position statement from the European psychiatric association (EPA), supported by the European association for the study of diabetes (EASD) and the European Society of cardiology (ESC). European Psychiatry 2009;24:412-24.

12 Colton CW, Manderscheid RW. Congruencies in increased mortality rates, years of potential life lost, and causes of death among public mental health clients in eight states. Prev Chronic Dis 2006;3:A42-A.

13 Vancampfort D, Stubbs B, Mitchell AJ, et al. Risk of metabolic syndrome and its components in people with schizophrenia and related psychotic disorders, bipolar disorder and major depressive disorder: a systematic review and meta-analysis. World Psychiatry 2015;14:339-47.

14 Vancampfort D, Correll CU, Galling B, et al. Diabetes mellitus in people with schizophrenia, bipolar disorder and major depressive disorder: a systematic review and large scale meta-analysis. World Psychiatry 2016;15:166-74.

15 Kisely S, Crowe E, Lawrence D. Cancer-Related mortality in people with mental illness. JAMA Psychiatry 2013;70:209-17.

16 Cunningham R, Sarfati D, Stanley J, et al. Cancer survival in the context of mental illness: a national cohort study. Gen Hosp Psychiatry 2015;37:501-6. 
17 Dickey B, Normand S-LT, Weiss RD, et al. Medical morbidity, mental illness, and substance use disorders. PS 2002;53:861-7.

18 Muntaner C, Eaton WW, Miech R, et al. Socioeconomic position and major mental disorders. Epidemiol Rev 2004;26:53-62.

19 Gardner-Sood P, Lally J, Smith S, et al. Cardiovascular risk factors and metabolic syndrome in people with established psychotic illnesses: baseline data from the impact randomized controlled trial. Psychol Med 2015;45:2619-29.

20 Daumit GL, Goldberg RW, Anthony C, et al. Physical activity patterns in adults with severe mental illness. J Nerv Ment Dis 2005;193:641-6.

21 Osborn DPJ, Nazareth I, King MB. Physical activity, dietary habits and coronary heart disease risk factor knowledge amongst people with severe mental illness. Soc Psychiatry Psychiatr Epidemiol 2007;42:787-93.

22 Margolese HC, Carlos Negrete J, Tempier R, et al. A 12-month prospective follow-up study of patients with schizophrenia-spectrum disorders and substance abuse: changes in psychiatric symptoms and substance use. Schizophr Res 2006;83:65-75.

23 Szatkowski L, McNeill A. Diverging trends in smoking behaviors according to mental health status. Nicotine Tob Res 2015;17:356-60.

24 Dickerson F, Stallings CR, Origoni AE, et al. Cigarette smoking among persons with schizophrenia or bipolar disorder in routine clinical settings, 1999-2011. PS 2013;64:44-50.

25 Lawrence D, Kisely S. Review: inequalities in healthcare provision for people with severe mental illness. J Psychopharmacol 2010;24:61-8.

26 Druss BG, von Esenwein SA. Improving general medical care for persons with mental and addictive disorders: systematic review. Gen Hosp Psychiatry 2006;28:145-53.

27 Mitchell AJ, Malone D, Doebbeling CC. Quality of medical care for people with and without comorbid mental illness and substance misuse: systematic review of comparative studies. Br J Psychiatry 2009;194:491-9.

28 van Boekel LC, Brouwers EPM, van Weeghel J, et al. Stigma among health professionals towards patients with substance use disorders and its consequences for healthcare delivery: systematic review. Drug Alcohol Depend 2013;131:23-35.

29 Happell B, Ewart SB, Bocking J, et al. 'That red flag on your file': misinterpreting physical symptoms as mental illness. J Clin Nurs 2016;25:2933-42.

30 Flanagan S, Damery S, Combes G. The effectiveness of integrated care interventions in improving patient quality of life (QOL) for patients with chronic conditions. An overview of the systematic review evidence. Health Qual Life Outcomes 2017;15.

31 Kodner D. All together now: a conceptual exploration of integrated care. Hcq 2009;13:6-15.

32 Zlateva I, Anderson D, Coman E, et al. Development and validation of the medical home care coordination survey for assessing care coordination in the primary care setting from the patient and provider perspectives. BMC Health Serv Res 2015;15.

33 World Health Organization Regional Office for Europe. Integrated care models: an overview (working document. Copenhagen, Denmark: World Health Organization Regional Office for Europe, 2016.

34 Singer SJ, Burgers J, Friedberg M, et al. Defining and measuring integrated patient care: promoting the next frontier in health care delivery. Med Care Res Rev 2011;68:112-27.
35 Rodgers M, Dalton J, Harden M, et al. Integrated care to address the physical health needs of people with severe mental illness: a mapping review of the recent evidence on barriers, facilitators and evaluations. Int J Integr Care 2018;18.

36 Bradford DW, Cunningham NT, Slubicki MN, et al. An evidence synthesis of care models to improve general medical outcomes for individuals with serious mental illness. J Clin Psychiatry 2013;74:e754-64.

37 Bartels SJ, Pratt SI, Mueser KT, et al. Integrated IMR for psychiatric and general medical illness for adults aged 50 or older with serious mental illness. PS 2014;65:330-7.

38 Happell B, Platania-Phung C, Scott D. Mental health nurse incentive program: facilitating physical health care for people with mental illness? Int J Ment Health Nurs 2013;22:399-408.

39 Nover $\mathrm{CH}$. Implementing a mental health and primary care partnership program in Placer County, California. Soc Work Health Care 2014;53:156-82.

40 Maki M, Bjorklund P. Improving cardiovascular disease screening in community mental health centers. Perspect Psychiatr Care 2013;49:179-86.

41 Kelly E, Fulginiti A, Pahwa R, et al. A pilot test of a peer navigator intervention for improving the health of individuals with serious mental illness. Community Ment Health J 2014;50:435-46.

42 Solomon P, Hanrahan NP, Hurford M, et al. Lessons learned from implementing a pilot RCT of transitional care model for individuals with serious mental illness. Arch Psychiatr Nurs 2014;28:250-5.

43 Grant BF, Stinson FS, Dawson DA, et al. Prevalence and cooccurrence of substance use disorders and independent mood and anxiety disorders: results from the National epidemiologic survey on alcohol and related conditions. Arch Gen Psychiatry 2004;61:807-16.

44 Regier DA, Farmer ME, Rae DS, et al. Comorbidity of mental disorders with alcohol and other drug abuse. results from the epidemiologic catchment area (ECA) study. JAMA 1990;264:2511-8.

45 Burnam MA, Watkins KE. Substance abuse with mental disorders: specialized public systems and integrated care. Health Aff 2006;25:648-58.

46 Tricco AC, Lillie E, Zarin W, et al. PRISMA extension for scoping reviews (PRISMA-ScR): checklist and explanation. Ann Intern Med 2018;169:467-73.

47 Daudt HML, van Mossel C, Scott SJ. Enhancing the scoping study methodology: a large, inter-professional team's experience with Arksey and O'Malley's framework. BMC Med Res Methodol 2013;13.

48 Arksey H, O'Malley L. Scoping studies: towards a methodological framework. Int J Soc Res Methodol 2005;8:19-32.

49 Levac D, Colquhoun H, O'Brien KK. Scoping studies: advancing the methodology. Implementation Sci 2010;5.

50 Peters MDJ, Godfrey CM, Khalil H, et al. Guidance for conducting systematic scoping reviews. Int J Evid Based Healthc 2015;13:141-6.

51 Kessler RC, Barker PR, Colpe LJ, et al. Screening for serious mental illness in the general population. Arch Gen Psychiatry 2003;60:184-9.

52 Kodner DL, Spreeuwenberg C. Integrated care: meaning, logic, applications, and implications - a discussion paper. Int J Integr Care 2002;2:e12. 